\title{
IDENTIFICATION IN ADDITIVE ERROR MODELS WITH DISCRETE ENDOGENOUS VARIABLES
}

Andrew Chesher

THE INSTITUTE FOR FISCAL STUDIES DEPARTMENT OF ECONOMICS, UCL 


\title{
Identification in additive error models with discrete endogenous variables
}

\author{
Andrew Chesher \\ Centre for Microdata Methods and Practice, IfS and UCL
}

September 5th 2004

\begin{abstract}
In additive error models with a discrete endogenous variable identification cannot be achieved under a marginal covariation condition when the support of instruments is sparse relative to the support of the endogenous variable. An iterated covariation condition with a weak montonicity restriction is shown to have set identifying power.
\end{abstract}

\section{INTRODUCTION}

In an additive error model: $Y_{1}=h\left(Y_{2}\right)+\varepsilon_{1}$ with a discrete endogenous variable $Y_{2}$, nonparametric identification of $h(\cdot)$ requires a vector of instruments $X$ to have at least as many points of support as the endogenous variable under the conventional marginal covariation condition: $E\left[\varepsilon_{1} \mid X=x\right]=c$, a constant invariant with respect to $x$.

Das (2004) and Florens and Malavolti (2003) study identification and estimation of $h(\cdot)$ under this condition. In practice this support condition may not be satisfied and there are additional difficulties when the number of points of support of $Y_{2}$ is unbounded.

This note explores the utility for identification of values yielded by $h(\cdot)$ of an iterated covariation condition, $E\left[\varepsilon_{1} \mid \varepsilon_{2}=e_{2} \cap X=x\right]=c\left(e_{2}\right)$ where $c(\cdot)$ is a function which does not depend on $x$ and $\varepsilon_{2}$ is a random variable which generates stochastic variation in $Y_{2}$ given $X$.

It is shown that, when the cardinality of the support of $X$ is at least equal to that of $Y_{2}$ the iterated condition has no additional power for identification of $h(\cdot)$ relative to the marginal covariation condition. However when the support of $X$ is deficient, if $c(\cdot)$ is restricted to be monotonic, then the iterated covariation condition can yield closed set identification of $h(\cdot)$ at particular points of support of $Y_{2}$ and, if instruments are not weak, of differences of $h(\cdot)$ across points of support.

\section{DeFinitions}

A discrete outcome $Y_{2}$ has $M$ known points of support $y_{2}^{1}<\cdots<y_{2}^{M}$ independent of a list of covariates $X$. For $m \in\{1, \ldots, M\}$ define $p^{m}(x) \equiv P\left[Y_{2} \leq y_{2}^{m} \mid X=x\right]$, the associated conditional quantile function:

$$
Q_{Y_{2} \mid X}(p \mid x)=y_{2}^{m} \text { where } m=\inf _{n \in\{1, \ldots, M\}}\left\{n: p^{n}(x) \geq p\right\}
$$

and the probability masses on the points of support of $Y_{2}: p_{m}(x) \equiv p^{m}(x)-p^{m-1}(x)=$ $P\left[Y_{2}=y_{2}^{m} \mid X=x\right]$ each restricted to lie in $(0,1)$ for all $x$.

A continuous outcome $Y_{1}$ and discrete outcome $Y_{2}$ are determined by the structural equations

$$
\begin{aligned}
Y_{1} & =h_{1}\left(Y_{2}\right)+\varepsilon_{1} \\
Y_{2} & =g\left(X, \varepsilon_{2}\right)
\end{aligned}
$$


where $\varepsilon_{1}$ and $\varepsilon_{2}$ are continuously distributed latent variates. ${ }^{1}$ The variate $\varepsilon_{2}$ is normalised $\operatorname{Unif}(0,1)$ and $g\left(x, e_{2}\right) \equiv Q_{Y_{2} \mid X}\left(e_{2} \mid x\right)$ is the conditional $e_{2}$-quantile function of $Y_{2}$ given $X$. Under this normalisation $Y_{2}$ has the conditional distribution function $p^{m}(x)$ given $X=x$.

\section{MARGINAL COVARIATION RESTRICTIONS}

Consider the following marginal covariation restriction as employed by Das (2004) and Florens and Malavolti (2003).

MC: For all $x, E\left[\varepsilon_{1} \mid X=x\right]=c$ where $c$ is invariant with respect to $x$.

Normalise $c=0$. Define $\mu(x) \equiv E\left[Y_{1} \mid X=x\right]$ and, for $m \in\{1, \ldots, M\}, h_{m} \equiv h\left(y_{2}^{m}\right)$, and $D_{m} \equiv 1\left[Y_{2}=y_{2}^{m}\right]$. Since $Y_{1}=\sum_{m=1}^{M} D_{m} h\left(y_{2}^{m}\right)+\varepsilon_{1}$ there is: $\mu(x)=\sum_{m=1}^{M} p_{m}(x) h_{m}$. Consider $N$ values of $X:\left\{x_{n}\right\}_{n=1}^{N}$. With the definitions below: $\mu_{N}=P_{N} \theta$.

$$
\mu_{N} \equiv\left[\begin{array}{c}
\mu\left(x_{1}\right) \\
\vdots \\
\mu\left(x_{N}\right)
\end{array}\right] \quad \theta \equiv\left[\begin{array}{c}
h_{1} \\
\vdots \\
h_{M}
\end{array}\right] \quad P_{N} \equiv\left[\begin{array}{ccc}
p_{1}\left(x_{1}\right) & \cdots & p_{M}\left(x_{1}\right) \\
\vdots & \ddots & \vdots \\
p_{1}\left(x_{N}\right) & \cdots & p_{M}\left(x_{N}\right)
\end{array}\right]
$$

The value of $\theta$ is identified without further restriction if and only if $\operatorname{rank}\left(P_{N}\right)=M$ for which a necessary condition is $N^{*} \geq M$ where $N^{*}$ is the number of distinct values in $\left\{x_{n}\right\}_{n=1}^{N}$. When the rank condition is satisfied there is for any $N \times N$ full rank matrix $W$,

$$
\theta=\left(P_{N}^{\prime} W^{-1} P_{N}\right)^{-1} P_{N}^{\prime} W^{-1} \mu_{N}
$$

which suggests analog GLS-type estimators with the elements of $P_{N}$ replaced by estimated conditional probabilities and the elements of $\mu_{N}$ replaced by values of $Y_{1}$. If $M$ is not finite there are clearly difficulties in implementing such procedures and even when $M$ is finite, if it is large then there may be extreme collinearity amongst the columns of $P_{N}$.

If the cardinality of the support of $X$ is less than that of the support of the discrete endogenous variable, then the value of $\theta$ is not identified. Further, since $p_{m}(x) \in(0,1)$ for all $x$, no element of $\theta$ is identified unless additional restrictions are introduced. The elements of $\theta$ are restricted to lie on $N^{*}$ hyperplanes in $\mathbb{R}^{M}$ so there is set identification of the value of $\theta$, but the set in which the value falls is not closed. ${ }^{2}$

\footnotetext{
${ }^{1}$ There are no covariates appearing in the structural function $h$ in equation (1). Covariates other than $X$ could appear, in which case, in this analysis of nonparametric identification, the arguments that follow will apply at each value of those covariates at which identification of a value of $h$ is sought. The covariates $X$ appearing in equation (2) could also be arguments of the structural function $h$ but its value must then be insensitive to the identifying variations in $X$ considered below.

${ }^{2}$ If there are the restrictions $R \theta=r$ where $R$ is a known $J \times M$ matrix and $r$ is a known $J$ element vector then $\theta$ is identified if and only if $\operatorname{rank}\left(\left[\begin{array}{ll}R^{\prime} & P_{N}^{\prime}\end{array}\right]\right)=M$ for which a necessary condition is $J+N^{*} \geq M$. One might entertain the restriction that $h$ is a $K$ th degree polynomial function of $Y_{2}$. Then, because suitably defined $(K+1)$ th differences of the elements of $\theta$ must be zero, there are $(M-1-K)$ linear restrictions on $\theta$. It follows that, if the covariate vector $X$ and the discrete endogenous variable have respectively $N^{*}$ and $M$ points of support with $N^{*}<M, \theta$ may be identified if $h$ is restricted to lie on a polynomial of degree $K$ where $K<N^{*}$. If $K \geq N^{*}$ then the restrictions may be overidentifying.
} 


\section{ITERATED COVARIATION CONDITION}

Now consider the following iterated covariation restriction.

IC: For all $x, E\left[\varepsilon_{1} \mid \varepsilon_{2}=e_{2} \cap X=x\right]=c\left(e_{2}\right) . E\left[\varepsilon_{1} \mid X=x\right]$ is finite.

Since $\varepsilon_{2}$ is independent of $X$ this implies the marginal covariation condition $\mathbf{M C}$ with $c \equiv \int_{0}^{1} c\left(e_{2}\right) d e_{2}$. However the iterated condition $\mathbf{I C}$ is not implied by MC. For example, for any function $a(x)$, if $E\left[\varepsilon_{1} \mid \varepsilon_{2}=e_{2} \cap X=x\right]=a(x)+2(c-a(x)) e_{2}$, which violates IC, then $E\left[\varepsilon_{1} \mid X=x\right]=c$ and $\mathbf{M C}$ is satisfied. So IC is more restrictive than MC. There is thus the possibility that it has greater identifying power, an issue explored in this Section.

When the cardinality of the support of $X$ is at least equal to the cardinality of the support of the discrete endogenous variable IC provides no additional identifying information for the structural function $h$. This is shown in Section 4.1.

Otherwise IC can yield closed set identification of values of the structural function if an additional restriction is imposed. This is shown in Section 4.2.

4.1. Rich support. For $z \in[0,1]$ define $C(z)=\int_{0}^{z} c(w) d w$. Clearly $C(0)=0$. Normalise $E\left[\varepsilon_{1} \mid X=x\right]=C(1)=0$.

The conditional expectation of $Y_{1}$ given $Y_{2}=y_{2}^{m}$ and $X=x$ is

$$
E\left(Y_{1} \mid Y_{2}=y_{2}^{m} \cap X=x\right)=h\left(y_{2}^{m}\right)+E\left(\varepsilon_{1} \mid \varepsilon_{2} \in\left(p^{m-1}(x), p^{m}(x)\right]\right)
$$

and since

$$
E\left(\varepsilon_{1} \mid \varepsilon_{2} \in\left(p^{m-1}(x), p^{m}(x)\right]\right)=\frac{1}{p_{m}(x)} \int_{p^{m-1}(x)}^{p^{m}(x)} c\left(e_{2}\right) d e_{2}
$$

there is:

$$
E\left(Y_{1} \mid Y_{2}=y_{2}^{m} \cap X=x\right)=h\left(y_{2}^{m}\right)+\frac{1}{p_{m}(x)}\left\{C\left(p^{m}(x)\right)-C\left(p^{m-1}(x)\right)\right\} .
$$

Suppose $M$ is finite and for $m \in\{1, \ldots, M\}$ and $N$ values of $X,\left\{x_{n}\right\}_{n=1}^{N}$ define the following terms.

$$
\begin{aligned}
\mu_{m n} & \equiv E\left(Y_{1} \mid Y_{2}=y_{2}^{m} \cap X=x_{n}\right) \\
p_{m n} & \equiv p_{m}\left(x_{n}\right) \equiv P\left[Y_{2}=y_{2}^{m} \mid X=x_{n}\right] \\
C_{n}^{m} & \equiv C\left(p^{m}\left(x_{n}\right)\right)
\end{aligned}
$$

There is, for $m \in\{1, \ldots, M\}$ and $n \in\{1, \ldots, N\}$

$$
\mu_{m n}=h_{m}+p_{m n}^{-1}\left(C_{n}^{m}-C_{n}^{m-1}\right)
$$

with, for all $n, C_{n}^{0}=0$ by definition, and $C_{n}^{M}=1$ by normalisation. ${ }^{3}$

Data are informative about the $2 \times M \times N$ values of the terms $\mu_{m n}$ and $p_{m n}$. The $M \times N$ equations (6) involve $M$ values of the terms $h_{m}$ and $(M+1) \times N$ values of the terms $C_{n}^{m}$ of which $2 \times N$ are determined by definition or normalisation. Therefore a necessary condition for the identification of the $M+(M-1) \times N$ undetermined values of the terms $h_{m}$ and $C_{n}^{m}$ is $M \times N \geq M+(M-1) \times N$, that is $N \geq M$, just as when the marginal covariation condition $\mathbf{M C}$ is imposed.

$$
{ }^{3} C_{n}^{M}=1 \text { because } p^{M}(x)=1 \text { for all } x .
$$


Summing the terms $\mu_{m n}$ across $m$ after multiplication by $p_{m n}$ there is, for each $n \in$ $\{1, \ldots, N\}$, in view of equation (6)

$$
\sum_{m=1}^{M} p_{m n} \mu_{m n}=\sum_{m=1}^{M} p_{m n} h_{m}=\mu\left(x_{n}\right)
$$

where $\mu\left(x_{n}\right)=E\left[Y_{1} \mid X=x_{n}\right] .{ }^{4}$ These equations are identical to the equations $\mu_{N}=P_{N} \theta$ obtained under MC and when $\operatorname{rank}\left(P_{N}\right)=M$ they can be solved for the $M$ unknown $h_{m}$ 's. A necessary condition is that $N^{*} \geq M$.

When the support of $X$ is rich in the sense that the cardinality of the support of $X$ is at least equal to the cardinality of the support of the discrete endogenous variable the conditions IC and $\mathbf{M C}$ lead to the same identifying correspondence for $\theta$. Under IC there is the possibility of identifying values of the terms $C_{n}^{m}$ which is not possible under MC. IC has no additional identifying power relative to $\mathbf{M C}$ for $\theta$ when the support of $X$ is rich.

When the support of $X$ is sparse in the sense that $N^{*}<M$ then $\mathbf{I C}$ can have additional closed set identifying power if a monotonicity restriction is imposed on the function $c(\cdot)$, as demonstrated in the next Section.

4.2. Sparse support. Impose the additional restriction that $c\left(e_{2}\right)$ is a monotonic function of $e_{2} \cdot{ }^{5}$ When $c$ is increasing there is then, in view of (4), the inequality

$$
c\left(p^{m-1}(x)\right) \leq E\left(\varepsilon_{1} \mid \varepsilon_{2} \in\left(p^{m-1}(x), p^{m}(x)\right]\right) \leq c\left(p^{m}(x)\right)
$$

which on adding $h\left(y_{2}^{m}\right)$ implies

$$
h\left(y_{2}^{m}\right)+c\left(p^{m-1}(x)\right) \leq E\left(Y_{1} \mid Y_{2}=y_{2}^{m} \cap X=x\right) \leq h\left(y_{2}^{m}\right)+c\left(p^{m}(x)\right)
$$

with the inequalities reversed when $c$ is decreasing. ${ }^{6}$

Suppose there exist values of $X,\left\{x_{m-1}, x_{m}\right\}$, such that for some $\bar{e}_{2} \in(0,1)$

$$
p^{m}\left(x_{m}\right) \leq \bar{e}_{2} \leq p^{m-1}\left(x_{m-1}\right)
$$

and consider the case in which $c$ is an increasing function. Since:

$$
c\left(p^{m}\left(x_{m}\right)\right) \leq c\left(\bar{e}_{2}\right) \leq c\left(p^{m-1}\left(x_{m-1}\right)\right)
$$

there are the following inequalities.

$$
\begin{aligned}
E\left(Y_{1} \mid Y_{2}=y_{2}^{m} \cap X=x_{m}\right) & \leq h\left(y_{2}^{m}\right)+c\left(p^{m}\left(x_{m}\right)\right) \\
& \leq h\left(y_{2}^{m}\right)+c\left(\bar{e}_{2}\right) \\
& \leq h\left(y_{2}^{m}\right)+c\left(p^{m-1}\left(x_{m-1}\right)\right) \\
& \leq E\left(Y_{1} \mid Y_{2}=y_{2}^{m} \cap X=x_{m-1}\right)
\end{aligned}
$$

\footnotetext{
${ }^{4}$ This expression results because $\sum_{m=0}^{M}\left(C_{n}^{m}-C_{n}^{m-1}\right)=C(1)=0$.

${ }^{5}$ This sort of montonicty restriction is imposed on conditional quantile functions in Chesher (2003) which studies identification in non-additive error models with discrete endogenous variables.

${ }^{6}$ If $c\left(e_{2}\right)=c$, constant for variations in $e_{2}$, in which case $\varepsilon_{1}$ is mean independent of $\varepsilon_{2}$, then for any $m$,

$$
E\left(Y_{1} \mid Y_{2}=y_{2}^{m}, X=x\right)=h\left(y_{2}^{m}\right)+c=E\left(Y_{1} \mid Y_{2}=y_{2}^{m}\right)
$$

and there is point identification of $h\left(y_{2}^{m}\right)$ at all values of $m$.
} 
The inequalities are reversed if $c$ is a decreasing function and the following inequality results.

$$
\begin{gathered}
\min \left(E\left(Y_{1} \mid Y_{2}=y_{2}^{m} \cap X=x_{m}\right), E\left(Y_{1} \mid Y_{2}=y_{2}^{m} \cap X=x_{m-1}\right)\right) \\
\leq h\left(y_{2}^{m}\right)+c\left(\bar{e}_{2}\right) \leq \\
\max \left(E\left(Y_{1} \mid Y_{2}=y_{2}^{m} \cap X=x_{m}\right), E\left(Y_{1} \mid Y_{2}=y_{2}^{m} \cap X=x_{m-1}\right)\right)
\end{gathered}
$$

This inequality holds at any point of support $m$ for which there exist $x_{m-1}$ and $x_{m}$ satisfying (7) any value of $\varepsilon_{2}, \bar{e}_{2}$. When this condition holds at many points of support for the same value $\bar{e}_{2}$ the value of the structural function is identified at those points of support up to a common location shift $c\left(\bar{e}_{2}\right)$ and then differences of the structural function, $h\left(y_{2}^{m}\right)-h\left(y_{2}^{n}\right)$ are identified.

The condition (7) cannot hold when $y_{2}^{m}$ is an extreme point of support carrying nonzero probability mass. For example when $y_{2}^{m}$ is the largest value achieved by $Y_{2},(7)$ can only hold for $\left\{x_{m-1}, x_{m}\right\}$ such that $p^{m}\left(x_{m}\right)=p^{m-1}\left(x_{m-1}\right)=1$ but then there is no probability mass on $y_{2}^{m}$.

The bounds are easily estimated using sample averages of $Y_{1}$ at $y_{2}^{m}$ and values of $X$ satisfying (7). In practice those values of $X$ will be determined using an estimate of the conditional distribution of $Y_{2}$ given $X$ which will have implications for the computation of standard errors.

\section{Strength of instruments And Support}

As noted in Section 3, under MC the identifiability of the structural function depends critically on the support of $X$. If $X$ has fewer points of support than the discrete endogenous variable then the value of the structural function is not identified at any point of support without further restriction.

Under IC and a monotonicity restriction on $c\left(e_{2}\right)$ the interval identifiability of values and differences of a structural function is greatly affected by the strength of the instruments but, with strong instruments, the support of $X$ does not play a critical role. With a strong enough instruments there can be interval identification of many values of a structural function up to common location shift even when the instruments have only two points of support. However when instruments are weak there may be no possibility of interval identification.

An example illustrating lack of identifiability is shown in Table 1 which exhibits conditional distribution functions for $Y_{2}$ with four points of support when $X$ has two points of support. In this case, because for all $m$,

$\max \left(P\left[Y_{2} \leq y_{2}^{m-1} \mid X=0\right], P\left[Y_{2} \leq y_{2}^{m-1} \mid X=1\right]\right)<\min \left(P\left[Y_{2} \leq y_{2}^{m} \mid X=0\right], P\left[Y_{2} \leq y_{2}^{m} \mid X=1\right]\right)$

there is no value of $\bar{e}_{2}$ and $m$ for which there are values of $X,\left\{x_{m-1}, x_{m}\right\}$ such that the condition (7) holds. Chesher (2003) shows that this situation prevails in the data used in Angrist and Krueger (1991).

Contrast this case with the one shown in Table 2. Here the effect of $X$ on the conditional distribution of $Y_{2}$ is substantial and there is interval identification of the value of the structural function at $Y_{2}=2$ and $Y_{2}=3$ up to a common location shift, even though $X$ has just two points of support. This case is now worked through in detail to illustrate the results of the Section 4.2. 
Table 1: Conditional distribution functions of $Y_{2}$ given $X=0$ and 1 - Example 1

\begin{tabular}{|c|c|c|}
\hline$y_{2}^{m}$ & $P\left[Y_{2} \leq y_{2}^{m} \mid X=0\right]=p^{m}(0)$ & $P\left[Y_{2} \leq y_{2}^{m} \mid X=1\right]=p^{m}(1)$ \\
\hline \hline 1 & 0.3 & 0.2 \\
\hline 2 & 0.5 & 0.4 \\
\hline 3 & 0.7 & 0.6 \\
\hline 4 & 1.0 & 1.0 \\
\hline
\end{tabular}

Table 2: Conditional distribution functions of $Y_{2}$ given $X=0$ and 1 - Example 2

\begin{tabular}{|c|c|c|}
\hline$y_{2}^{m}$ & $P\left[Y_{2} \leq y_{2}^{m} \mid X=0\right]=p^{m}(0)$ & $P\left[Y_{2} \leq y_{2}^{m} \mid X=1\right]=p^{m}(1)$ \\
\hline \hline 1 & 0.7 & 0.1 \\
\hline 2 & 0.8 & 0.2 \\
\hline 3 & 0.9 & 0.3 \\
\hline 4 & 1.0 & 1.0 \\
\hline
\end{tabular}

Since in Table 2:

$$
\begin{array}{lcll}
\forall & \bar{e}_{2} \in(0.2,0.7): & p^{2}(1) \leq \bar{e}_{2} \leq p^{1}(0) \\
\forall & \bar{e}_{2} \in(0.3,0.8): & p^{3}(1) \leq \bar{e}_{2} \leq p^{2}(0)
\end{array}
$$

there is, if $c(\cdot)$ is increasing,

$$
\begin{array}{lccccc}
\forall & \bar{e}_{2} & \in(0.2,0.7): & E\left[Y_{1} \mid Y_{2}=2 \cap X=1\right] \leq h(2)+c\left(\bar{e}_{2}\right) \leq E\left[Y_{1} \mid Y_{2}=2 \cap X=0\right] \\
\forall & \bar{e}_{2} \in(0.3,0.8): & E\left[Y_{1} \mid Y_{2}=3 \cap X=1\right] \leq h(3)+c\left(\bar{e}_{2}\right) \leq E\left[Y_{1} \mid Y_{2}=3 \cap X=0\right]
\end{array}
$$

with the inequalities reversed if $c(\cdot)$ is decreasing. The intervals over which these two inequalities hold both include the interval $(0.3,0.7)$ and so $h(2)$ and $h(3)$ are interval identified up to the common location shift $c\left(\bar{e}_{2}\right)$ for all $\bar{e}_{2}$ in this common interval. As a result there is interval identification of the partial difference $h(2)-h(3)$, as follows

$$
\begin{gathered}
E\left[Y_{1} \mid Y_{2}=2 \cap X=1\right]-E\left[Y_{1} \mid Y_{2}=3 \cap X=0\right] \\
\leq h(2)-h(3) \leq \\
E\left[Y_{1} \mid Y_{2}=2 \cap X=0\right]-E\left[Y_{1} \mid Y_{2}=3 \cap X=1\right]
\end{gathered}
$$

with the inequalities reversed if $c\left(e_{2}\right)$ is decreasing.

It is instructive to take this example a little further. Suppose that the data generating process has $\varepsilon_{1} \mid\left(\varepsilon_{2}, X\right) \sim N\left(c\left(\varepsilon_{2}\right), \sigma^{2}\right)$ and $c\left(\varepsilon_{2}\right)=\lambda_{0}+\lambda_{1} \Phi^{-1}\left(\varepsilon_{2}\right)$ where $\Phi^{-1}$ is the standard normal quantile (inverse distribution) function. Since $\varepsilon_{2} \sim \operatorname{Unif}(0,1)$, $\Phi^{-1}\left(\varepsilon_{2}\right) \sim N(0,1)$ and $c\left(\varepsilon_{2}\right) \sim N\left(\lambda_{0}, \lambda_{1}^{2}\right)$, so in this data generating process $\varepsilon_{1}$ and $c\left(\varepsilon_{2}\right)$ are jointly normally distributed. There is, for $m \in\{1,2,3,4\}$ and $x \in\{0,1\}$ :

$$
\begin{aligned}
E\left[Y_{1} \mid Y_{2}=y_{2}^{m} \cap X=x\right] & =h\left(y_{2}^{m}\right)+\lambda_{0}+\frac{\lambda_{1}}{p_{m}(x)} \int_{p^{m-1}(x)}^{p^{m}(x)} \Phi^{-1}\left(e_{2}\right) d e_{2} \\
& =h\left(y_{2}^{m}\right)+\lambda_{0}+\frac{\lambda_{1}}{p_{m}(x)}\left(\phi\left(\Phi^{-1}\left(p^{m-1}(x)\right)\right)-\phi\left(\Phi^{-1}\left(p^{m}(x)\right)\right)\right)
\end{aligned}
$$

where $\phi$ denotes the standard normal density function. 
Using the values in Table 2 there is:

$$
\begin{aligned}
& E\left[Y_{1} \mid Y_{2}=2 \cap X=1\right]=h(2)+\lambda_{0}-1.045 \lambda_{1} \\
& E\left[Y_{1} \mid Y_{2}=2 \cap X=0\right]=h(2)+\lambda_{0}+0.677 \lambda_{1} \\
& E\left[Y_{1} \mid Y_{2}=3 \cap X=1\right]=h(3)+\lambda_{0}-0.677 \lambda_{1} \\
& E\left[Y_{1} \mid Y_{2}=3 \cap X=0\right]=h(3)+\lambda_{0}+1.045 \lambda_{1}
\end{aligned}
$$

and the absolute value of the width of the bounds (8) is $3.444 \times \lambda_{1}$. As $\lambda_{1} \rightarrow 0$, that is as $\varepsilon_{1}$ and $\varepsilon_{2}$ approach independence and $Y_{2}$ becomes exogenous, the width of the bounds falls to zero and point identification is secured.

\section{REFERENCES}

Angrist, Joshua, D., And Alan B. Krueger (1991): "Does compulsory schooling attendance affect schooling and earnings?" Quarterly Journal of Economics, 106, 979 1014.

Chesher, Andrew (2003): "Nonparametric identification under discrete variation," Centre for Microdata Methods and Practice Working Paper CWP 19/03.

Das, Mitali (2004): "Instrumental Variables Estimation of Nonparametric Models with Discrete Endogenous Variables," forthcoming, Journal of Econometrics.

Florens, Jean-Pierre and Laetitia Malavolti (2003): "Instrumental Regression with Discrete Variables," presented at the 2003 European Meeting of the Econometric Society, Stockholm. 